\title{
From reference model selection to controller validation: Application to Loewner Data-Driven Control
}

\author{
Pauline Kergus $^{1 \star}$, Martine Olivi ${ }^{2}$, Charles Poussot-Vassal ${ }^{1}$ and Fabrice Demourant ${ }^{1}$
}

\begin{abstract}
The choice of a reference model in data-driven control techniques is a critical step. Indeed, it should represent the desired closed-loop performances and be achievable by the plant at the same time. In this paper, we propose a method to build such a reference model, both reproducible by the system and having a desired behaviour. It is applicable to Linear TimeInvariant (LTI) monovariable systems and relies on the estimation of the plant's instabilities through a data-driven stability analysis technique. The L-DDC (Loewner Data Driven Control) algorithm is used to illustrate the impact of the choice of the reference model on the control design process. Finally, the proposed choice of specifications allows to use a controller validation technique based on the small-gain theorem.
\end{abstract}

Keywords: data-driven control, interpolation method, Loewner, stability

\section{INTRODUCTION}

$\mathbf{F}$ OR many applications, a mathematical description of the system, derived from physical laws, is not available. In this case, the controller has to be designed on the basis of experimental measurements. The first solution consists in identifying a model of the plant and then using any kind of model-based technique to obtain a control law (indirect methods). It is indicated for problems where a reliable model with bounded modeling errors is available. On the other side, the data-driven strategy directly computes the controller from the experimental data. Such techniques are also called direct methods and may be appealing in cases where such a controloriented model is too time-consuming, too complex or too costly to obtain. The two strategies, model-based and datadriven, are complementary in sense that they do not address the same categories of problem.

\section{A. The data-driven model-reference problem}

Numerous direct methods have been proposed to try to achieve the best possible performance without using any plant model. Among them, some methods, like Correlation-basedTuning (CbT, [1]), Virtual Reference Feedback Tuning (VRFT, [2]) or Loewner Data-Driven Control (L-DDC, [3]), can be designated as model-reference techniques. The principle of the model-reference problem is recalled on Figure 1. These approaches only require data from the plant $P,\left\{\omega_{i}, P\left(\imath \omega_{i}\right)\right\}_{i=1}^{N}$,

Thanks to the whole FACTAS team at INRIA and particularly to Adam Cooman for his precious advice on data-driven stability analysis.

${ }^{1}$ ONERA, DTIS, Information Processing and Systems, Toulouse, France

${ }^{2}$ FACTAS team, INRIA Sophia Antipolis, France

${ }^{\star}$ Corresponding author: pauline.kergus@onera.fr

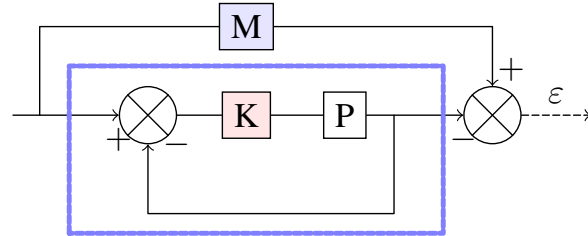

Fig. 1: Problem formulation: $M$ is the desired closed-loop, $P$ is the plant and $K$ the controller to be designed.

and the desired closed-loop behaviour, given as a reference model transfer function $M \in \mathcal{R} \mathcal{H}_{\infty}$. The objective is to design a controller that minimizes the error $\epsilon$ between the resulting closed-loop and the desired one $M$. These three techniques rely on the concept of ideal controller $K^{\star}$ : the controller that would give exactly the desired closed-loop behaviour. By definition, in the frequency-domain, it is given by (1).

$$
K^{\star}\left(\imath \omega_{i}\right)=\left(P\left(\imath \omega_{i}\right)-P\left(\imath \omega_{i}\right) M\left(\imath \omega_{i}\right)\right)^{-1} M\left(\imath \omega_{i}\right) .
$$

The obtained controller $K$ is then supposed to be as close as possible to this ideal controller $K^{\star}$. To this aim, the $\mathrm{CbT}$ and the VRFT minimize a reference tracking control objective over a set $\mathcal{K}$ of linearly parametrized controllers. The problem is made convex by assuming $K^{\star} \in \mathcal{K}$. This is a strong assumption and makes the choice of the structure of the controller a critical step. The L-DDC algorithm avoids this step by identifying a linear rational and time invariant controller model $K$ of the ideal controller $K^{\star}$.

However, as explained in [4], whenever the plant is NonMinimum Phase (NMP), the ideal controller might compensate the Right-Half-Plane (RHP) zeros of the plant. According to (1), this is determined by the choice of the reference model $M$. This is why, despite the apparent simplicity of this type of techniques, the choice of the model-reference $M$ is a critical step, as it is pointed out in [4], [5] and [6]. The difficulty of choosing the reference model resides in the fact that it should not only represent a desired closed-loop behaviour, but also an achievable one, given the plant to be controlled. The behaviours that a plant can reach are determined by its instabilities.

Different ways to choose a reference model have been proposed. In the case of the VRFT, [4] and [6] have proposed to parametrize the reference model function and to find a good one along with the design of the controller. However, the case of unstable plants is not tackled by this technique. In [7], it is proposed to define the reference model $M$ according to the nature of the system and through a stable filter that will 
determine the performances. The filter is applied to functions that are already known to be achievable.It should be noted that no solution has been proposed for plants that are both non-minimum phase and unstable.

\section{B. Overview of the contributions and structure of the paper}

The objective of this paper is to propose a technique to construct an achievable reference model $M$ that can be used for data-driven control purposes. The proposed method is based on frequency-domain data. It relies on a data-driven instability detection technique introduced in [8]. Then, once the instabilities of the plant are detected, it is possible to build an achievable reference model. The L-DDC method can then be applied. Finally, the data-driven validation of the controller, based on [7], is applied in order to check that the obtained controller stabilizes the plant internally.

This article is organized in seven sections. Section II illustrates the impact of the choice of the reference model by applying the L-DDC method on a NMP plant. The conditions for a reference model to be reachable by a SISO (Single Input - Single Output) plant will also be expressed. In Section III, the data-driven instabilities estimation introduced in [8] is recalled. The contribution of this paper, which is the choice of an achievable reference model $M$, is exposed in Section IV. The data-driven controller validation introduced in [7] is recalled in Section V. The proposed technique is applied in Section VI on the same numerical example than in Section II, in order to analyze the impact of the proposed reference model choice. Finally, conclusions and outlooks are given in Section VII.

\section{Notations}

$\imath$ denotes the complex variable, $(.)^{T}$ is the transpose of a matrix and (. $)^{\dagger}$ its pseudo-inverse. $\mathcal{L}_{\infty}$ is the set of functions bounded on the imaginary axis, $\mathcal{H}_{\infty}$ is the subset of $\mathcal{L}_{\infty}$ containing the functions with an analytic continuation in the RHP. $\mathcal{R H}_{\infty}$ is the real rational subset of $\mathcal{H}_{\infty} \cdot \mathcal{L}_{2}$ is the set of square integrable functions. $\mathcal{H}_{2}$ is the subspace of $\mathcal{L}_{2}$ of stable functions while $\overline{\mathcal{H}}_{2}$, the orthogonal of $\mathcal{H}_{2}$, is the subspace of $\mathcal{L}_{2}$ of anti-stable functions.

\section{PROBLEM FORMULATION}

\section{A. Preliminary results: Loewner Data-Driven Control}

The L-DDC algorithm, introduced in [3], is based on frequency-domain data $\left\{\omega_{i}, P\left(\imath \omega_{i}\right)\right\}_{i=1}^{N}$ collected from the plant. It is a one shot technique. The order of the controller is a tunable parameter. It only requires a reference model $M$. The L-DDC algorithm is recalled in Algorithm 1.

The Loewner framework allows to identify an interpolating model $K$ of the ideal controller $K^{\star}$ in the second step, see [9]. In order to avoid the compensation of the plant's RHP zeros, the L-DDC alorithm has been modified in [10]: the interpolating model $K$ is projected on $\mathcal{R} \mathcal{H}_{\infty}$, using the technique presented in [11]. It corresponds to step 3 of Algorithm 1. The reduction has also been modified in order to be more robust to noisy data. This is possible through a residue-based implementation instead of the classic SVD approach, see [12] for more information.

The identification of the ideal controller in the second step makes it unnecessary to choose a structure a priori for the controller, contrary to CbT or VRFT. Despite this strength, the L-DDC technique, as the CbT and the VRFT, is confronted to the problem of choosing an achievable reference model for an unknown plant.

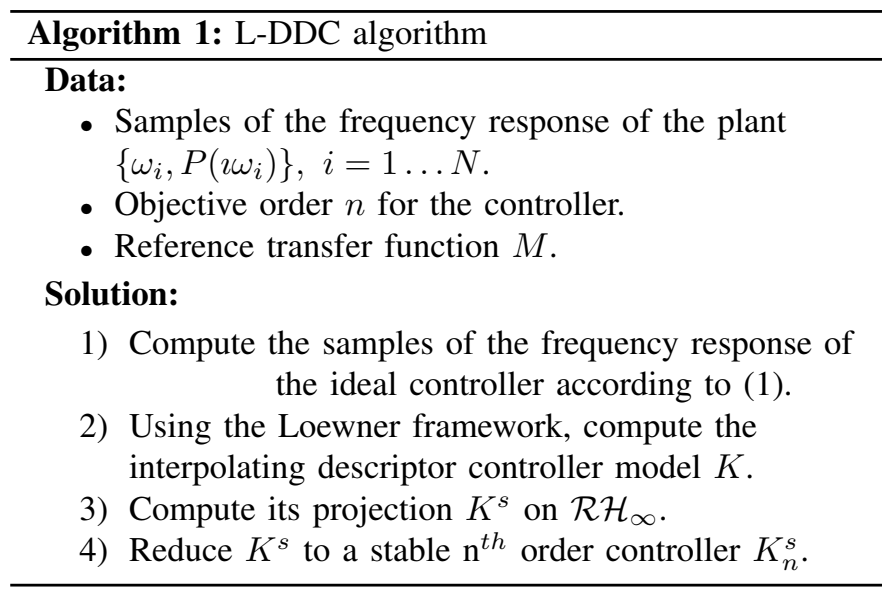

\section{B. Application to a non-minimum phase plant}

In order to illustrate the difficulty of choosing $M$, the LDDC algorithm is applied on a non-minimum phase plant. The system is a flexible transmission benchmark introduced in [13]. Here, the unloaded case is considered. The system is described by the following transfer function:

$$
P(s)=\frac{0.03616(s-140.5)(s-40)^{3}}{\left(s^{2}+1.071 s+157.9\right)\left(s^{2}+3.172 s+1936\right)} .
$$

This continuous-time model is obtained by applying a bilinear transform on the original discrete-time one given in [13].

Remark 1: This simple LTI plant is not representative of the class of the systems for which it would be preferable to use a data-driven control technique. It is used along this paper for mere illustation purposes. This example has been used as a benchmark for various control techniques, model-based or data-driven. Other examples are available in [14].

The plant has four RHP zeros that will limit the performances of the controlled system. The data is noise-free and $N=200$ points $\left\{\omega_{i}, P\left(\imath \omega_{i}\right)\right\}_{i=1 \ldots N}$, logspaced between 1 and $10^{3}$ rad.s ${ }^{-1}$ are used. The reference model $M$ is a second order stable transfer function:

$$
M(s)=\frac{k}{\frac{1}{\omega_{0}^{2}} s^{2}+\frac{2 \xi}{\omega_{0}} s+1}
$$

with $\omega_{0}=10 \mathrm{rad} . \mathrm{s}^{-1}, \xi=1$ and $k=1$. The exact expression of $K^{\star}$ is given by (4) and can be obtained through an exact Loewner based interpolation:

$$
K^{\star}(s)=k \frac{\left(s^{2}+1.071 s+157.9\right)\left(s^{2}+3.172 s+1936\right)}{s(s+20)(s-140.5)(s-40)^{3}},
$$


with $k=2.7655 \times 10^{5}$. Since the controller compensates the RHP zeros of the plant, the resulting closed-loop is internally unstable. It should be noted that this problem is almost impossible to detect in a data-driven framework since the model of the plant, given in (2) here, is supposed to be unknown.

To avoid the compensation of instabilities in the openloop, a stable controller $K^{s} \in \mathcal{R} \mathcal{H}_{\infty}$ is obtained in [10], see Algorithm 1. In this case, enforcing the stability of the controller degrades the closed-loop performances. This is due to the fact that a stable controller model cannot reflect the behaviour of $K^{\star}$. This is called the mismatched case [4]: $K^{\star}$ does not belong to the chosen class of controllers, which is $\mathcal{R H} \mathcal{H}_{\infty}$ here. The same issue exists when using CbT or VRFT.

\section{Finding an achievable reference model}

For this reason, choosing an appropriate reference model $M$ is a critical step for all data-driven techniques based on this paradigm. Indeed, the associated ideal controller $K^{\star}$ should stabilize the plant internally. This is the case if and only if $S=\left(1+K^{\star} P\right)^{-1}$ is stable and if there are no compensation of instabilities between the ideal controller $K^{\star}$ and the plant $P$, see [15]. Since $S=1-M, S \in \mathcal{R H}_{\infty}$ is equivalent to $M \in \mathcal{R H}_{\infty}$. The absence of instabilities compensation in the open-loop can be expressed as interpolatory conditions on $S$, and consequently on the reference model $M$. Therefore, when $P$ is a SISO plant, the closed-loop on Figure 1 is internally stable if and only if $M \in \mathcal{R H} \mathcal{H}_{\infty}$ and satisfies:

$$
\left\{\begin{array}{l}
\forall i=1 \ldots n_{z}, M\left(z_{i}\right)=0 \\
\forall j=1 \ldots n_{p}, M\left(p_{j}\right)=1
\end{array},\right.
$$

where $\left\{z_{i}\right\}_{i=1}^{n_{z}}$ and $\left\{p_{j}\right\}_{j=1}^{n_{p}}$ are respectively the unstable zeros and poles of the plant, which are assumed to be distinct. For MIMO (Multi-Inputs Multi-Outputs) plants, (5) becomes a tangential interpolation problem, see [16], which could be dealt with using the Loewner framework for example.

Remark 2: When the plant has multiple RHP poles or zeros, derivative constraints must be added. Any transfer function $\mathbf{M} \in \mathcal{R} \mathcal{H}_{\infty}$ that satisfies (6) is achievable by the plant:

$$
\left\{\begin{array}{l}
M\left(z_{i}\right)=M^{(1)}\left(z_{i}\right)=\cdots=M^{\left(m_{z_{i}}-1\right)}\left(z_{i}\right)=0 \\
M\left(p_{j}\right)=M^{(1)}\left(p_{j}\right)=\cdots=M^{\left(m_{p_{j}}-1\right)}\left(p_{j}\right)=1
\end{array},\right.
$$

where $m_{z_{i}}$ and $m_{p_{j}}$ denote the multiplicity of $z_{i}$ and $p_{j}$ respectively.

To sum up, the plant's instabilities, its RHP poles and zeros, are the main limitations of the possible control performances. Therefore, it is not possible for a plant to achieve a reference model that does not take them into account. In this case, the ideal controller $K^{\star}$ destabilizes the plant internally and represents a bad behaviour that should not be identified. In this paper, we propose to build a stable reference model that satisfies (5). First, the plant's instabilities are estimated using the method proposed in [8], recalled in the next section. Then the construction of the reference model is proposed in Section IV and applied on the example of the flexible transmission in Section VI.

\section{DATA-DRIVEN DETECTION OF INSTABILITIES}

The method proposed in [17] allows to detect and to estimate instabilities of a system on the basis of frequency-domain data only. It is then perfectly adapted to find a good reference $M$ for data-driven control purposes. It is implemented in the PISA toolbox [18]. This technique is recalled in Algorithm 2.

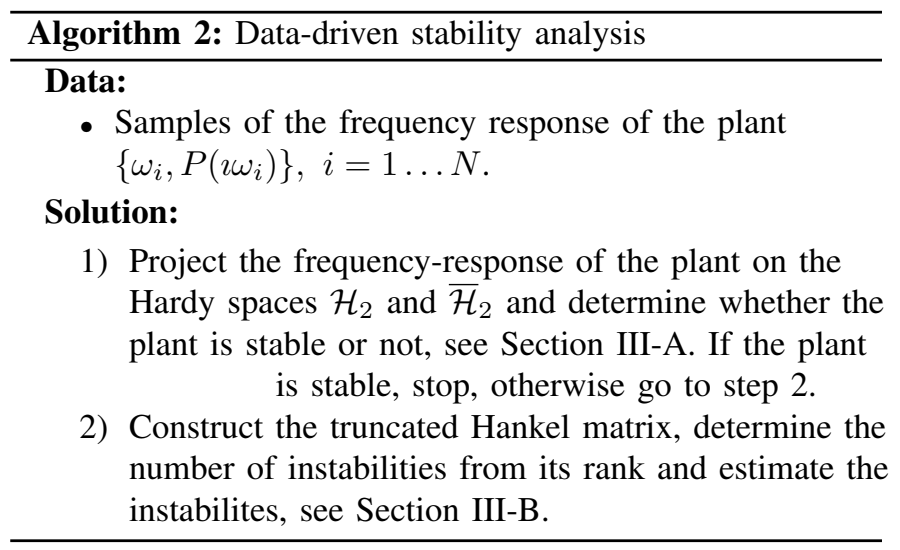

Remark 3: By applying this technique on the frequency response $P(\imath \omega)^{-1}$, one can determine if the plant is NMP and estimate the eventual RHP zeros of the plant $P$.

Remark 4: One could argue that interpolating the frequencyresponse of the plant would be much more straightforward to determine the poles and zeros of the plant, including the RHP ones. As explained in [17], over or undermodelling can lead to the idendification of non-physical instabilities. Therefore, it would consists not only in an interpolation of the data, but in a plant identification step, and the method would not be data-driven anymore. Indeed, data-driven control addresses the cases where model order selection is complicated for control purposes, but also for stability analysis.

\section{A. Stable and unstable projection of frequency-domain data}

In the first place, it is necessary to project the given frequency-response in order to detect the presence of instabilities. It is assumed that $P(\imath \omega) \in \mathcal{L}_{2}$, meaning that it can be written as follows: $P(\imath \omega)=P^{s}(\imath \omega)+P^{a s}(\imath \omega)$. $P^{s}$ is its stable projection and belongs to the Hardy space $\mathcal{H}_{2}$, while $P^{a s}$ is its anti-stable part, belonging to the orthogonal $\overline{\mathcal{H}}_{2}$.

First, the frequency response of the plant is moved to the unit circle so the projection boils down to a Fourier transform. The obtained frequency response is filtered to avoid edge effect during the projection: the sudden ending of the data would appear in the unstable part. These two steps are technical and the reader should refer to [17] for more insights. The stable and anti-stable projections of the plant's data are then obtained by computing the Fourier coefficients $\left\{c_{k}\right\}_{k \in \mathbb{Z}}$ :

$$
P^{s}(z)=\sum_{k=0}^{\infty} c_{k} z^{k} \quad \text { and } \quad P^{a s}(z)=\sum_{k=1}^{\infty} c_{-k} z^{-k} .
$$

A numerically efficient way to compute a given number $N_{F}$ of Fourier coefficients is the Fast Fourier Transform. To this aim, the plant's data is interpolated using a basic linear interpolation technique. 
If $P^{s}$ fits the frequency response measurements of the plant, it can be assumed that the plant is stable. Otherwise, the plant is unstable.

\section{B. Estimation of the location of the unstable poles}

The estimation of instabilities proposed in [8] is based on the Principal Hankel Components (PHC) method.It relies on the Hankel matrix containing the Fourier coefficients corresponding to the anti-stable projection $P^{a s}$. In practice, a truncation $\Phi_{n_{F}}$ of the infinite dimensionnal matrix $\Phi$ is used, see (8).

$$
\forall i, j=1 \ldots n_{F},\left[\Phi_{n_{F}}\right]_{i, j}=c_{-(i+j-1)}
$$

For a rational system with $n_{p}$ poles, the Hankel matrix $\Phi_{n_{F}}$ is of rank $n_{p}$. In order to find the number of poles of $P^{a s}$, which correspond to the unstable poles of $P$, a SVD is performed on the truncated Hankel matrix $\Phi_{n_{F}}$ :

$$
\Phi_{n_{F}}=\left(\begin{array}{ll}
U_{P} & U_{R}
\end{array}\right)\left(\begin{array}{cc}
S_{P} & 0 \\
0 & S_{R}
\end{array}\right)\left(\begin{array}{c}
V_{P}^{T} \\
V_{R}^{T}
\end{array}\right),
$$

with $S_{P} \in \mathbb{R}^{n_{p} \times n_{p}}$ containing the $n_{p}$ largest singular values, $U_{P} \in \mathbb{R}^{n_{F} \times n_{p}}$ and $V_{P} \in \mathbb{R}^{n_{F} \times n_{p}}$. The number of poles $n_{p}$ is the number of non-zero singular values in (9). The value of the corresponding RHP poles can be deduced from the truncated Hankel matrix, see [8].

\section{CONSTRUCTION OF THE REFERENCE MODEL}

Now that the instabilities of the plant are estimated, it is possible to build a stable reference model that gives the desired performances and satisfies (5). In order for the proposed method to stay user-friendly, the achievable reference model $M_{f}$ is obtained by filtering the initial one $M$. As a matter of fact, the easiest way for the user to express closed-loop performance specifications is to define a stable second-order model-reference through three parameters $\left\{\omega_{0}, \xi, k\right\}$, as in (3).

First, let us introduce the Blaschke products $B_{z}$ and $B_{p}$ defined respectively by the estimated RHP zeros $\left\{z_{i}\right\}_{i=1 \ldots n_{z}}$ and poles $\left\{p_{j}\right\}_{j=1 \ldots n_{p}}$ of the plant:

$$
B_{z}(s)=\prod_{i=1}^{n_{z}} \frac{s-z_{i}}{s+z_{i}} \quad \text { and } \quad B_{p}(s)=\prod_{j=1}^{n_{p}} \frac{s-p_{j}}{s+p_{j}} .
$$

These two functions, $B_{z}$ and $B_{p}$, are stable. The choice of a stable reference model $M_{f}$ that satisfies the interpolatory conditons (5) is done as follows:

1) If the plant is stable and minimum phase: any stable and minimum-phase $M$ specified by the user is achievable by the plant.

2) If the plant is stable and non-minimum phase: $M_{f}=$ $M B_{z}$ with $M$ stable is achievable by the plant.

3) If the plant is unstable and minimum phase: $M_{f}=$ $1-(1-M) B_{p}$ with $M$ stable is achievable by the plant.

4) If the plant is unstable and non-minimum phase: $M_{f}=M B_{z} F$ with $M$ stable is achievable by the plant, where the filter $F$ is defined as follows: $F(s)=$ $\frac{\sum_{k=1}^{n_{p}} \gamma_{k} l_{k}(s)}{\prod_{j=1}^{n}\left(s+p_{j}\right)}$, with $\gamma_{k}=\frac{\prod_{j=1}^{n_{p}}\left(p_{k}+p_{j}\right)}{M\left(p_{k}\right) B_{z}\left(p_{k}\right)}$ and $l_{k}(s)=$ $\prod_{j=1, j \neq k}^{n_{p}} \frac{s-p_{j}}{p_{k}-p_{j}}$, for $k=1 \ldots n_{p}$.
Proof 1: In case 1, for a stable and minimum phase plant $P$ and a stable model reference $M$, the functions $(1-M)$ and $P(1-M)$ are also stable. Finally, according to the definition of the ideal controller, $K^{\star}(1-M)=M P^{-1}$ is also stable. Therefore, $K^{\star}$ stabilizes the plant internally.

For other cases, $K^{\star}$ stabilizes the plant internally if and only if $M_{f}$ is stable and satisfies (5). In case 2 and 3, this is obviously the case since, by definition, $B_{z}$ and $B_{p}$ are stable and satisfy $\forall i, B_{z}\left(z_{i}\right)=0$ and $\forall j, B_{p}\left(p_{j}\right)=0$.

In case 4 , the use of $B_{z}$ ensures that, for any RHP zeros $z_{i}$, $M_{f}\left(z_{i}\right)=0$. The additional filter $F$ is stable and is supposed to ensure that, for any RHP poles $p_{j}, M_{f}\left(p_{j}\right)=1$. It is defined using Lagrange polynomials which satisfies $l_{k}\left(p_{j}\right)=\delta_{k, j}$. Consequently, $M_{f}$ satisfies (5).

Remark 5: Concerning the application of the proposed method to unstable plants, it should be noted that measurements from such plants are only accessible through simulations, as in [14], or by performing an experiment with an initial stabilizing controller.

Remark 6: In case 2 or 3, the multiplicity of the instabilities can be taken into account to satisfy the derivative constraints of (6) by taking:

$B_{z}(s)=\prod_{i=1}^{n_{z}}\left(\frac{s-z_{i}}{s+z_{i}}\right)^{m_{z_{i}}}$ and $B_{p}(s)=\prod_{j=1}^{n_{p}}\left(\frac{s-p_{j}}{s+p_{j}}\right)^{m_{p_{j}}}$.

Solving (6) is more complicated in case 4, where the Loewner framework could be used with derivative constraints in order to find an achievable reference model.

\section{DATA-DRIVEN CONTROLLER VALIDATION}

Now that an achievable reference model $M_{f}$ is built, the controller $K$ can be designed using the L-DDC algorithm [10]. Since the identified model may be a reduced-order one of the ideal controller $K^{\star}$, there is no guarantee in the design process that $K$ actually stabilizes the plant internally. In [7], a stability test was introduced, giving a sufficient condition for the final controller $K$ to stabilize the plant internally. It relies on the controller error $K-K^{\star}$. This validation technique has been applied to controllers obtained with the VRFT in [7]. It has been used as a stability constraint in the non-iterative CbT algorithm to enforce closed-loop stability in [19].

The trick to analyze closed-loop stability is to re-write the closed-loop according to the controller error $K-K^{\star}$ as shown on Figure 3. This scheme makes the controller error appear as a perturbation. It is then possible to obtain (11) as a sufficient closed-loop stability condition.

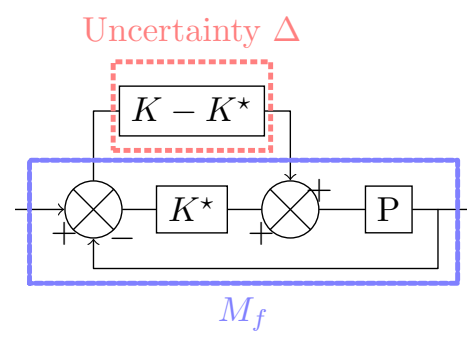

Fig. 3: Closed-loop interconnection scheme using the controller error. 


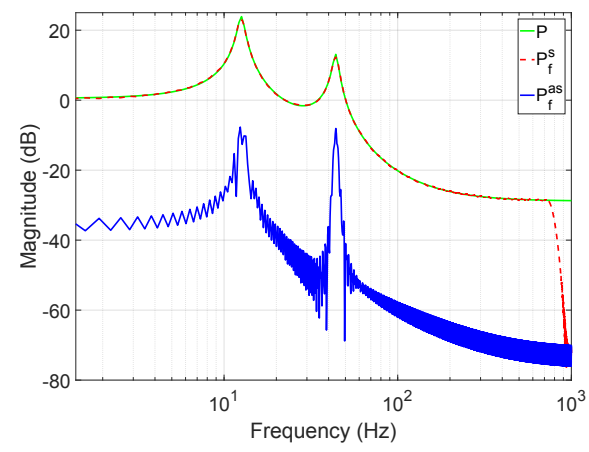

(a) Projection of $\left\{\omega_{i}, P\left(\imath \omega_{i}\right)\right\}_{i=1 \ldots N}$.

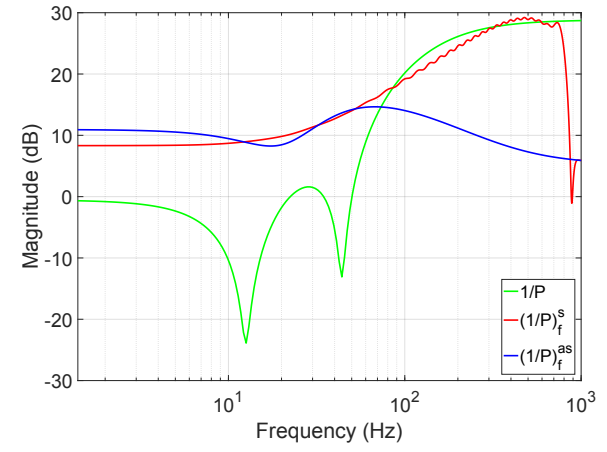

(b) Projection of $\left\{\omega_{i}, P^{-1}\left(\imath \omega_{i}\right)\right\}_{i=1}^{N}$.

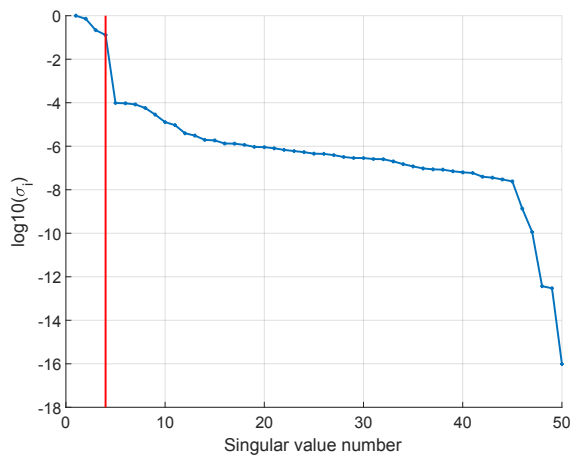

(c) Singular Value Decomposition of the Hankel matrix $\Phi$ : detection of four RHP zeros.

Fig. 2: Data-driven stability analysis of the flexible transmission example.

$$
\lambda_{0}=\left\|\frac{\left(K-K^{\star}\right) P}{1+K^{\star} P}\right\|_{\infty}=\left\|K P\left(1-M_{f}\right)-M_{f}\right\|_{\infty}<1 .
$$

The conditions to apply the small-gain theorem are the following: i) $\Delta=K-K^{\star}$ and ii) $P\left(1-M_{f}\right)$ should be stable. Thanks to the design of the reference model $M_{f}$ in Section IV, $K^{\star}$ stabilizes the plant internally, so $P\left(1-M_{f}\right)$ is stable. By construction of $M_{f}$, the ideal controller itself is also stable, see (1). Finally, since the L-DDC algorithm [10] enforces the stability of the identified controller $K, \Delta$ is stable. The sufficient condition (11) is obtained by applying the small-gain theorem, see [15].

Considering the dynamical system $\Lambda=K P\left(1-M_{f}\right)-M_{f}$, the stability test consists in estimating its $\mathcal{H}_{\infty}$-norm. Since the frequency response of $P$ is only known on a discrete frequency grid, so is the frequency response of $\Lambda$. The sufficient condition of (11) can be adapted as follows:

$$
\hat{\lambda}_{0}=\max _{i=1 \ldots N}\left|\Lambda\left(\imath \omega_{i}\right)\right|<1-\varepsilon,
$$

where $\varepsilon>0$ is a tolerance set by the user to avoid false positive results.

\section{ApplicATION}

Let us come back to the application of the flexible transmission introduced in Section II. The first step to design an achievable reference model $M_{f}$ is to conduct a data-driven stability analysis as explained in Section III-A. The results are visible on Figures $2 \mathrm{a}$ and $2 \mathrm{~b}$.

From Figure $2 \mathrm{a}$, it is clear that the plant is stable since the frequency response of $P_{f}^{s}$ fits the frequency response of the plant. According to Figure $2 \mathrm{~b}$, it seems that $P^{-1}$ is unstable for two reasons: its stable projection does not fit the data of $P^{-1}$ and its anti-stable projection contributes to the frequency response of $P^{-1}$. Therefore the plant $P$ is non-minimum phase.

The next step then consists in looking for the plant's RHP zeros by estimating the unstable poles of the frequency response $\left\{\omega_{i}, P^{-1}\left(\imath \omega_{i}\right)\right\}_{i=1}^{N}$ as explained in Section III-B.
The SVD of the Hankel matrix $\Phi$ defined in (8) is visible on Figure 2c. There are four singular values before the sharp drop, so it is chosen to estimate four instabilities. The estimated RHP zeros of the plant along with its real RHP zeros are given in Table I.

\begin{tabular}{|c|c|c|c|c|}
\hline Real RHP zeros of system (2) & 140.5 & 40 & 40 & 40 \\
\hline Estimated RHP zeros & 140.58 & $41.3-2 \imath$ & $41.3+2 \imath$ & 37.4 \\
\hline
\end{tabular}

TABLE I: Estimation of the RHP zeros of the plant.

Since the plant is stable but non-minimum phase, this application enters in case 2 from Section IV. $B_{z}$ is defined as in (10) and an achievable reference model is therefore $M_{f}=M B_{z}$. The identification of the controller is shown on Figure 4a.

Since the instabilities are not estimated exactly, the Loewner interpolation gives an unstable minimal realisation of order 10 of the ideal controller $K^{\star}$. However, by projecting the obtained controller model on $\mathcal{R} \mathcal{H}_{\infty}$, we obtain $K_{s}^{\star}$ with almost the same frequency response than $K^{\star}$. It means that the unstable poles of the minimal realisation $K^{\star}$ of the controller, which compensates the real unstable zeros of the plant, are almost equal to the estimated RHP zeros of the system. It is then possible to assimilate the minimal realisation $K^{\star}$ of the ideal controller to its stable projection $K_{s}^{\star}$ since $\left\|K^{\star}-K_{s}^{\star}\right\|_{\infty}=$ $8.10^{-4}$. Then the controller is reduced to a sixth-order model $K_{6}$, see (13).

Finally, the stability analysis detailed in Section $\mathrm{V}$ is applied. The $\mathcal{H}_{\infty}$-norm of $\Lambda$ is estimated through (12): $\hat{\lambda}_{0}=$ 0.0087. Therefore, since $\hat{\lambda}_{0}<1$ and according to (11), the obtained controller $K$ stabilizes the plant internally. The step response of the resulting closed-loop is visible on Figure $4 \mathrm{~b}$. We can see that the designed reference model $M_{f}$ reproduces the undershoot which is characteristic of the non-minimum phase behaviour of the plant: it means that the RHP zeros cannot be compensated and shows the intrinsic limitations of performances due to the plant's instabilities.

Remark 7: To illustrate the impact of the reference model selection on data-driven control techniques, the controller was only reduced to an order 6 to fit the ideal controller's data. For information, the reduced-order controller $K_{2}$ of order $n=2$ 


$$
K_{6}(s)=\frac{0.0423\left(s+6.604 .10^{4}\right)\left(s^{2}+1.418 s+160.8\right)\left(s^{2}+3.606 s+1954\right)}{s(s+148.9)\left(s^{2}+19.98 s+485.7\right)\left(s^{2}+112 s+4538\right)} \quad K_{2}(s)=\frac{-0.2911 s+199.6}{s(s+328.2)}
$$

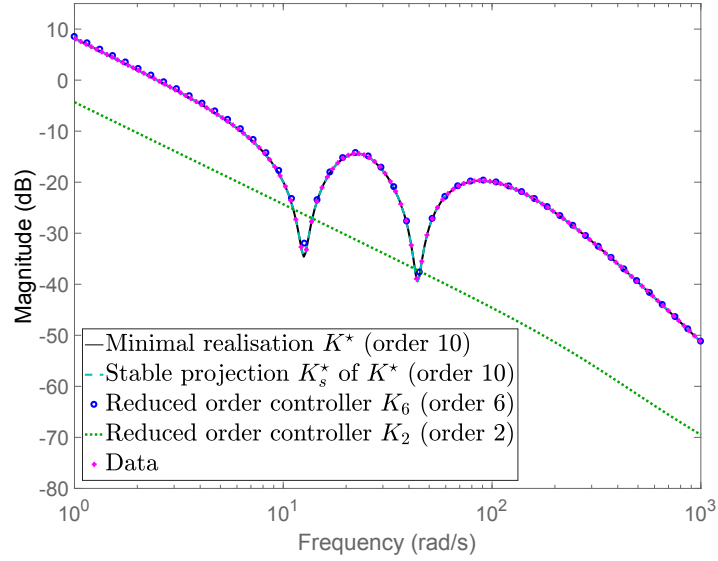

(a) Identification of the controller for an achievable reference model

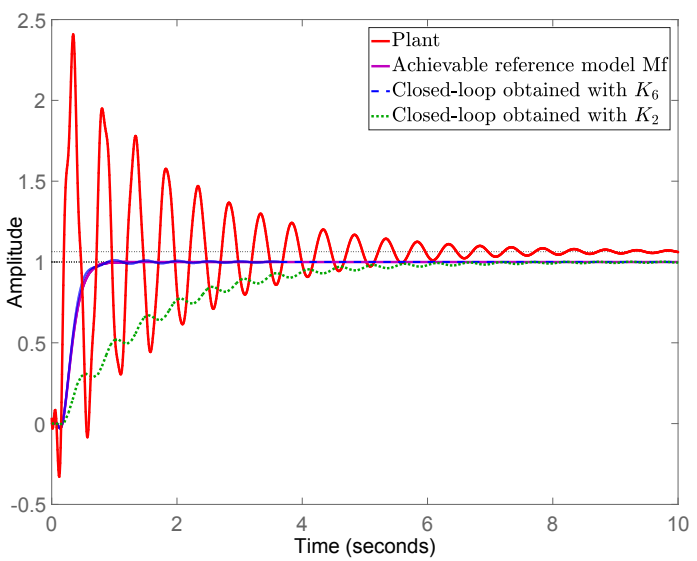

(b) Step response of the plant, of the achievable reference model and of the obtained closed-loop.

Fig. 4: Results of the L-DDC algorithm when using an achievable reference model on the flexible transmission benchmark.

is given in (13). Its frequency response is given on Figure 4a and the closed-loop performances are shown on Figure $4 \mathrm{~b}$.

\section{CONCLUSIONS}

In this paper, a novel approach to design an achievable model-reference for data-driven control techniques such as the VRFT, CbT or L-DDC, has been proposed. It relies on a data-driven estimation of the plant's instabilities. They are then used as interpolatory conditions to define the behaviors that the plant can reach. This technique is applicable to LTI SISO plants. The preliminary analysis of the plant's frequency response allows to design the specifications according to the nature of the system in a data-driven way. The proposed choice of reference model allows to meet the assumptions needed to use the stability criterion introduced in [7] based on the smallgain theorem. Therefore, it is possible to validate the obtained controller in terms of internal stability thanks to this sufficient condition.

The future of this work is to extend the choice of an achievable reference model to MIMO plants. To that extent, the framework developed in [16] to resolve the sensitivity minimization problem can be used. It would allow to give the interpolatory conditions defining an achievable multivariable model-reference. Another outlook would be to improve the design of $M_{f}$ when the system is both unstable and nonminimum phase in order to have a better control on the desired closed-loop performances.

\section{REFERENCES}

[1] A. Karimi, L. Miskovic, and D. Bonvin. Convergence analysis of an iterative correlation-based controller tuning method. In 15th IFAC World Congress, 2002.

[2] MC. Campi, A. Lecchini, and SM. Savaresi. Virtual reference feedback tuning: a direct method for the design of feedback controllers. Automatica, 2002.

[3] P. Kergus, C. Poussot-Vassal, F. Demourant, and S. Formentin Frequency-domain data-driven control design in the Loewner framework. 20th IFAC World Congress, 2017.
[4] AS. Bazanella, L. Campestrini, and D. Eckhard. Data-driven controller design: the H2 approach. Springer Science \& Business Media, 2011.

[5] D. Piga, S. Formentin, and A. Bemporad. Direct data-driven control of constrained systems. IEEE Transactions on Control Systems Technology, 2018.

[6] D. Selvi, D. Piga, and A. Bemporad. Towards direct data-driven modelfree design of optimal controllers. In European Control Conference. IEEE, 2018.

[7] K. van Heusden, A. Karimi, and D. Bonvin. Data-driven controller validation. In Proceedings of the 15th IFAC Symposium on System Identification, 2009.

[8] A. Cooman, F. Seyfert, and S. Amari. Estimating unstable poles in simulations of microwave circuits. In IEEE/MTT-S International Microwave Symposium, 2018.

[9] AC. Antoulas, S. Lefteriu, and AC. Ionita. A tutorial introduction to the Loewner framework for model reduction. Model Reduction and Approximation for Complex Systems, Birkhäuser, ISNM Series, 2015.

[10] P. Kergus, S. Formentin, C. Poussot-Vassal, and F. Demourant. Datadriven control design in the Loewner framework: Dealing with stability and noise. European Control Conference, 2018.

[11] M. Köhler. On the closest stable descriptor system in the respective spaces $\mathcal{R H}_{2}$ and $\mathcal{R} \mathcal{H}_{\infty}$. Linear Algebra and its Applications, 2014.

[12] S. Lefteriu, A.C. Ionita, and A.C. Antoulas. Modeling systems based on noisy frequency and time domain measurements. Perspectives in Mathematical System Theory, Control, and Signal Processing, 2010.

[13] ID. Landau, D. Rey, A. Karimi, A. Voda, and A. Franco. A flexible transmission system as a benchmark for robust digital control. European Journal of Control, 1995.

[14] P. Kergus, M. Olivi, C. Poussot-Vassal, and F. Demourant. Data-driven reference model selection and application to L-DDC design. arXiv preprint arXiv:1905.04003, 2019.

[15] K. Zhou and JC. Doyle. Essentials of robust control. Prentice Hall, 1998.

[16] K. Havre and S. Skogestad. Achievable performance of multivariable systems with unstable zeros and poles. International Journal of Control, 2001.

[17] A. Cooman, F. Seyfert, M. Olivi, S. Chevillard, and L. Baratchart. Model-free closed-loop stability analysis: A linear functional approach. IEEE Transactions on Microwave Theory and Techniques, 2018.

[18] PISA Toolbox for Circuit Stability Analysis, 2018. INRIA SophiaAntipolis, https://project.inria.fr/pisa/.

[19] K. Van Heusden, A. Karimi, and D. Bonvin. Data-driven model reference control with asymptotically guaranteed stability. International Journal of Adaptive Control and Signal Processing, 2011. 